MATEC Web of Conferences 11,01041 (2014)

DOI: $10.1051 /$ matecconf / 20141101041

(C) Owned by the authors, published by EDP Sciences, 2014

\title{
Variation de l'épaisseur du placage de déroulage des bois et son influence sur les caractéristiques mécaniques des panneaux LVL
}

\author{
Abdelhakim DAOUI1, 2, Clément Descamps3, Abdellatif ZERIZER2, Rémy MARCHAL3, Bartosz Pałubicki4 \\ ${ }^{1}$ Département Sciences techniques, Faculté des Sciences, Université M'hamed Bougara Boumerdes, Algérie \\ ${ }^{2}$ UR-MPE Unité de Recherche Matériaux Procédé Environnement, Université Bougara, Boumerdès, Algérie \\ ${ }^{3}$ Laboratoire Bourguignon des Matériaux et Procédés (LaBoMaP),ParisTech- ENSAM, Cluny, France \\ ${ }^{4}$ Dept. of Woodworking Machinery and Basis of Machines Construction, Poznan University, Poland
}

\begin{abstract}
Résumé. Le LVL (Laminated Veneer Lumber) est un matériau industriel très en vogue dans la construction, c'est un matériau dérivé du bois très apprécié dans la construction en ossature bois pour la faible dispersion de ses remarquables caractéristiques mécaniques, notamment en matière des contraintes axiales, pouvant aller jusqu'au double d'un bois massif.
\end{abstract}

Mots Clés: CND, fissuration, la colle, épaisseur du placage, propriétés mécaniques, LVL, déroulage.

\section{Présentation du travail réalisé}

Afin de faire varier le paramètre fissuration, le déroulage s'est effectué suivant plusieurs modalités faisant intervenir des facteurs d'influence notables sur la fissuration cyclique (épaisseur de placage, température d'étuvage et présence de la barre de pression lors du déroulage). Des panneaux ont été encollés et des éprouvettes en ont été extraites. Celles-ci ont été testées mécaniquement selon plusieurs méthodes et types de sollicitations.

\section{Matériels et méthodes}

\section{Echantillonnage}

Cet arbre de hêtre a grandi dans la forêt domaniale des Etangs, située à Saint Loup Géange, Saône et Loire, Bourgogne. La grume ( $13 \mathrm{~m}$ de long et $0,45 \mathrm{~m}$ de diamètre moyen) a été tronçonnée en 19 billons de 0,6m, numérotés de 1 à 19 en partant de la bille de pied.

\section{Etuvage}

Avant le déroulage, tous les billons ont été chauffés durant $24 \mathrm{~h} \pm 1 \mathrm{~h}$, soit à $20^{\circ} \mathrm{C}$, soit à $70^{\circ} \mathrm{C}$ par immersion dans une bouilloteuse thermorégulée à $\pm 1^{\circ} \mathrm{C}$.

\section{Déroulage}

Cette opération s'est effectuée sur la dérouleuse industrielle instrumentée SEM Automation du LaboMap (Cluny France). La vitesse de coupe fixée a été de 1,5m/s et l'angle de dépouille de $+1^{\circ}$. Lorsque la barre de pression est active, les côtes horizontales et verticales font respectivement $90 \%$ et $30 \%$ de l'épaisseur de déroulage. Nous avons produit du placage de trois épaisseurs $(1,3$ et $5 \mathrm{~mm})$, puis se sont ajouté de plus fortes épaisseurs (6 et $7 \mathrm{~mm}$ ).

Tableau Modalités de déroulage.

\begin{tabular}{|c|c|c|c|c|c|c|c|c|c|c|c|c|c|c|c|}
\hline Numéro de billon & 14 & 4 & 15 & 13 & 4 & 14 & 19 & 17 & 3 & 9 & 8 & 18 & 16 & 11 & 7 \\
\hline $\begin{array}{c}\text { Epaisseur de placage } \\
{[\mathrm{mm}]}\end{array}$ & 1 & 3 & 5 & 1 & 3 & 5 & 1 & 3 & 5 & 6 & 7 & 1 & 3 & 5 & 7 \\
\hline $\begin{array}{c}\text { Température d'étuvage } \\
200^{\circ} \mathrm{C}\end{array}$ & \multicolumn{1}{|c|}{$70^{\circ} \mathrm{C}$} \\
\hline Barre de pression (BP) & \multicolumn{3}{|c|}{ Sans BP } & \multicolumn{1}{c|}{ Avec BP } & \multicolumn{3}{c|}{ Sans BP } & \multicolumn{3}{c|}{ Avec BP } \\
\hline
\end{tabular}

\section{Mesure de la fissuration}

Plusieurs techniques pour estimer la fissuration d'un placage existent, comme la méthode par ultrasons proposée par [TOMPPO_2008].Dans notre cas, nous avons choisi celle développée dans le LaBoMap par 


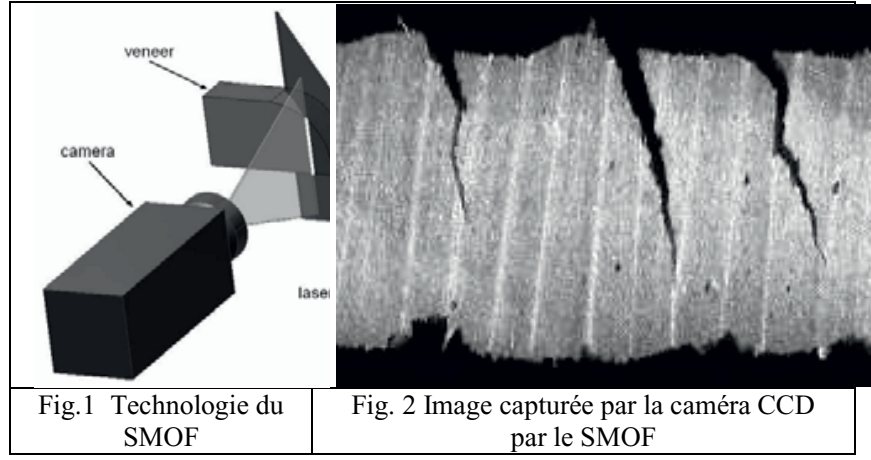

[PALUBICKI_2009] : le SMOF® (Système de Mesure Optique de la Fissuration). Cet outil détecte les fissures avec une caméra CCD linéaire $(36 \mathrm{KHz})$ et un capteur de déplacement laser (LDS) (Fig.1). L'image obtenue (Fig.2) est analysée par un programme développé sous LabView.

Sur l'image, il suffit de sélectionner le fond de chaque fissure (Fig. 3) pour obtenir un fichier .xls comprenant toutes les positions (Fig. 4). Ainsi, en connaissant la position « haute » du placage, son épaisseur et l'échelle de l'image, nous calculons les valeurs moyennes de profondeur et d'intervalles des fissures.

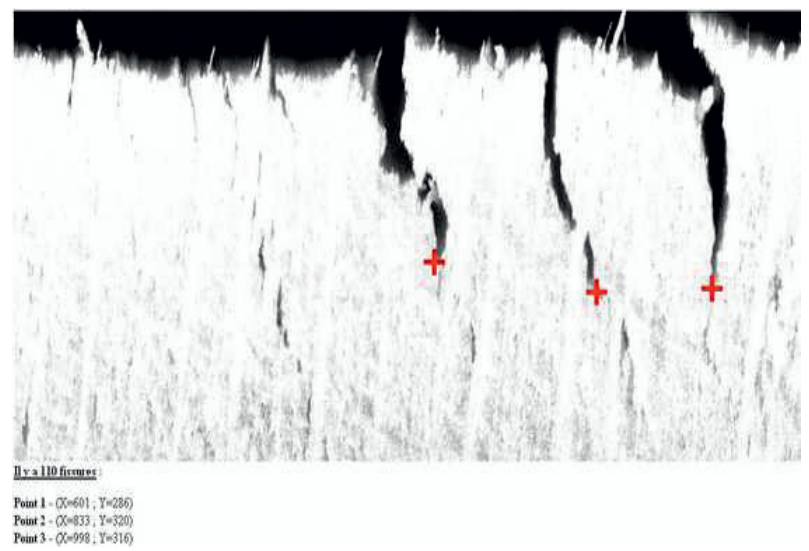

Fig.3 sélection des fissures

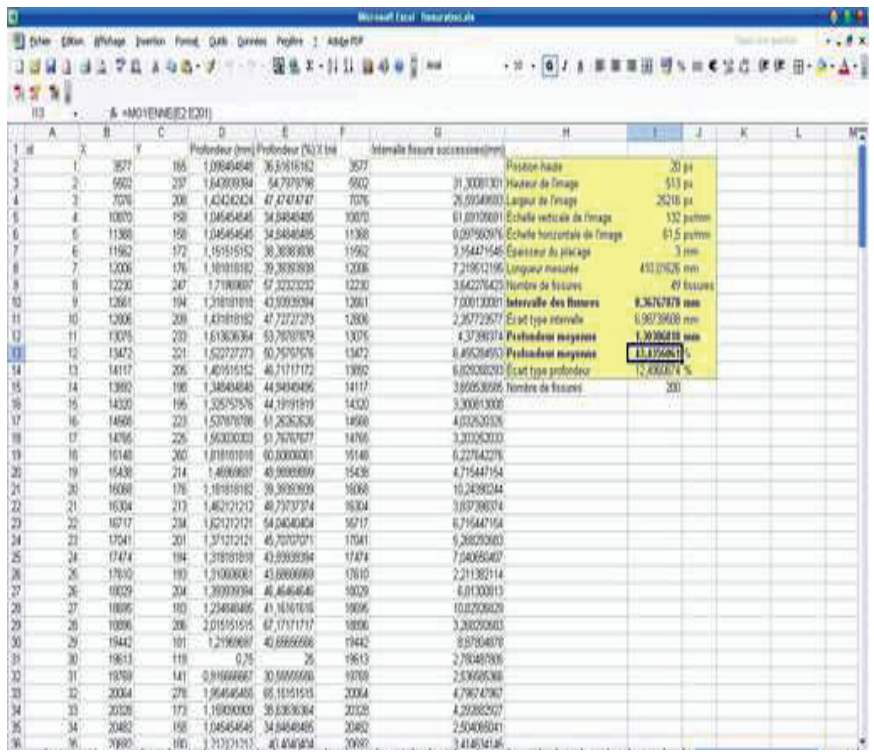

Fig. 4- Position des fissures

\section{Résultats et discussion}

Lorsqu'un placage a un état de surface très dégradé, on doit augmenter le grammage afin que la colle qui pénètre dans les aspérités du bois puisse tout de même recouvrir une surface suffisante pour assurer un collage correct. Etant donné que la qualité de cet état de surface diminue lorsqu'on augmente l'épaisseur, on parvient tout de même à diminuer de façon importante la proportion globale de colle dans le panneau (fig.5) en diminuant le nombre de plis (et donc de joints de colle).

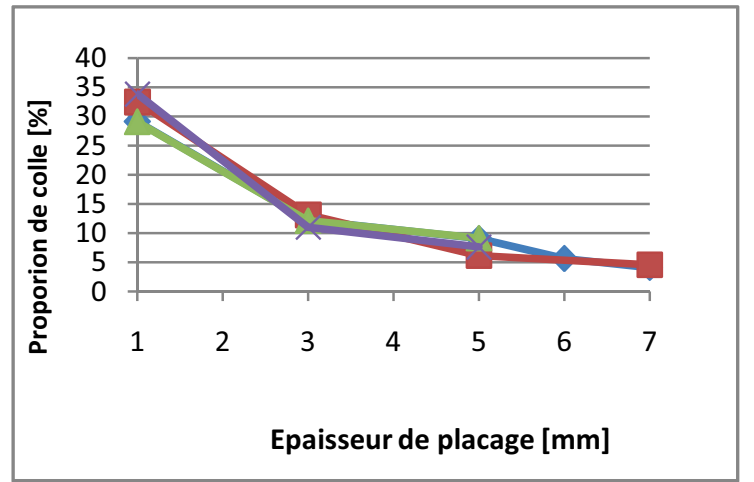

Fig.5 Evolution de la proportion de colle dans le panneau en fonction de l'épaisseur de déroulage 

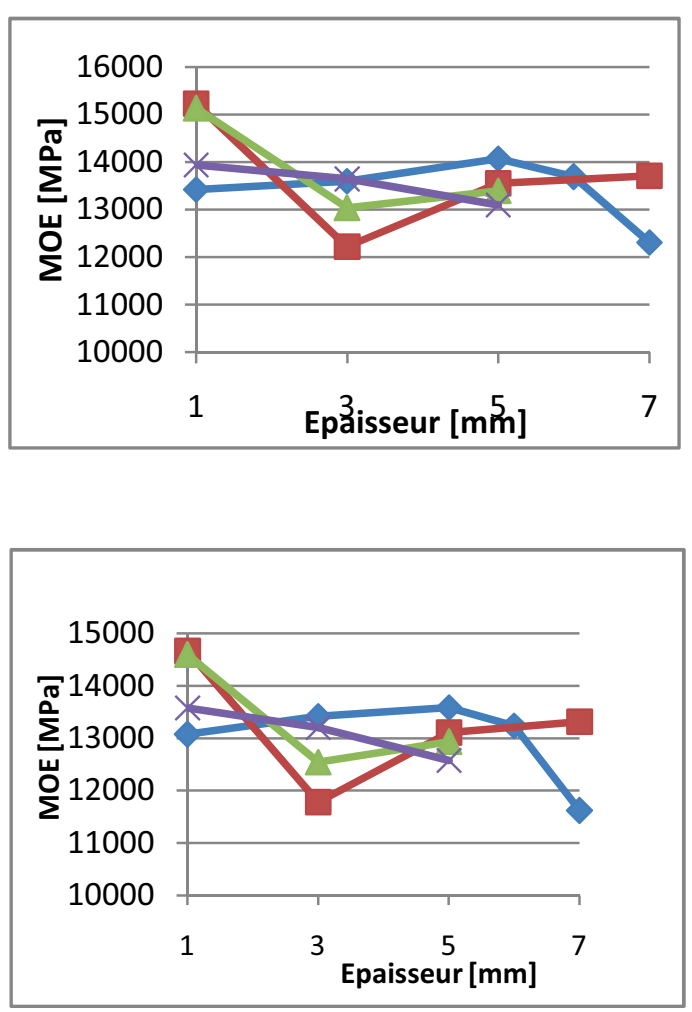

Fig. 6 Evolution de MOE en fonction de l'épaisseur en sollicitation à Plat selon Timoshenko \& Bernoulli
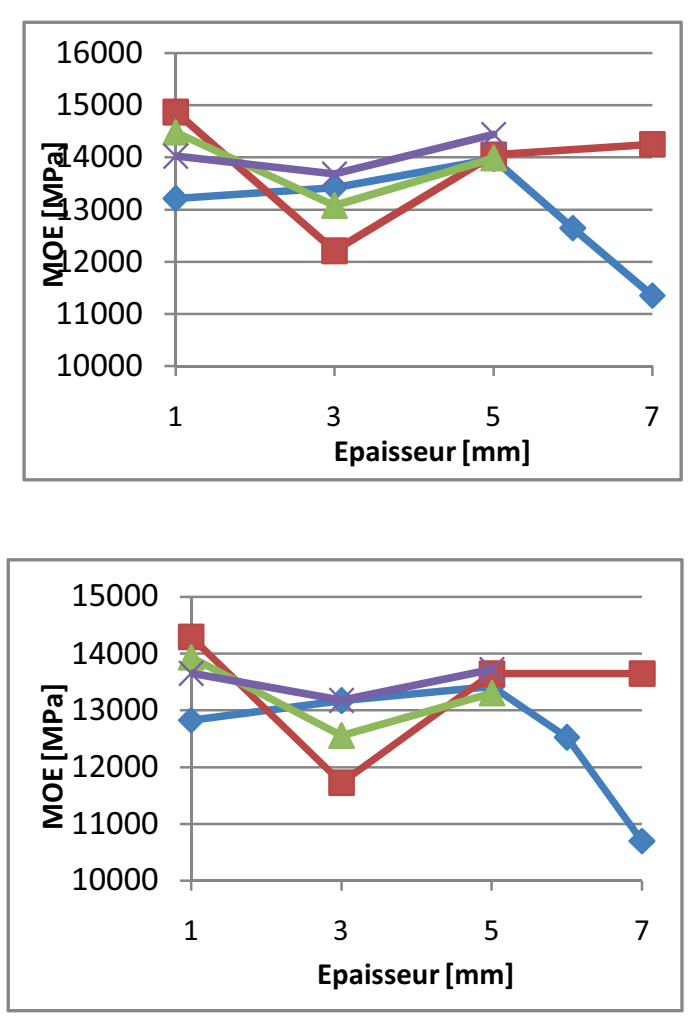

Fig. 7 Evolution de MOE en fonction de l'épaisseur en sollicitation à Plat selon Timoshenko \& Bernoulli
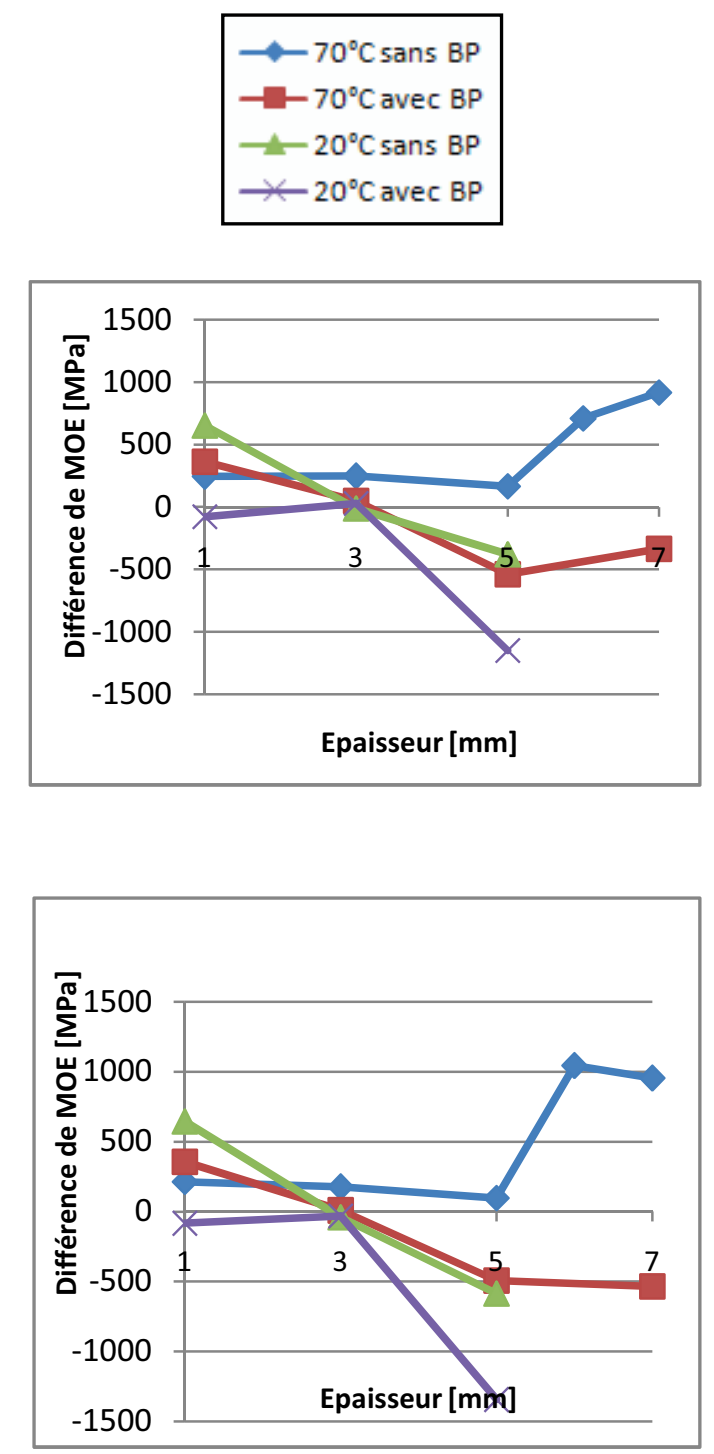

Fig. 8 Evolution de la différence de module de MOE entre sollicitation sur chant et à plat en fonction de l'épaisseur selon les modèles de Timoshenko et de Bernoulli

De ces graphes semble également se dégager une influence de la barre de pression et de la température d'étuvage qui ont un effet positif sur le module d'élasticité et la contrainte de rupture. On peut aussi ajouter qu'en chauffant le billon avant déroulage et en utilisant la barre de pression, on arrive à maintenir une résistance très correcte dans le LVL.

Quant à la différence entre la sollicitation à plat et sur chant (Fig. 8). Il semblerait qu'à partir d'une certaine épaisseur, les éprouvettes sont plus résistantes lorsque l'effort est appliqué à plat et il serait favorable de changer l'orientation des panneaux dans certains cas. 


\section{Conclusion}

Il faut savoir que cette étude ne représente qu'une première étape du programme d'optimisation du bilan écologique du LVL qui a pour objectif d'abord de chercher à diminuer l'utilisation de colle et par la suite employer des adhésifs « écologiques ».

On a pu en tirer qu'en augmentant l'épaisseur de déroulage, on diminue très fortement la proportion globale de colle par panneau tout en gardant des caractéristiques mécaniques correctes, à condition d'utiliser des conditions de coupe optimales et un traitement thermique préliminaire du billon.

Quant à l'influence fissuration, Les caractéristiques (fréquence et profondeur) de ces fissures peuvent être améliorées grâce aux conditions de coupe grâce à un chauffage du bois et à une utilisation d'une barre de pression lors du déroulage.

Toutefois, en faisant une régression linéaire toutes modalités confondues, on voit que la tendance globale des caractéristiques mécaniques est à la diminution lorsqu'on augmente l'épaisseur.

\section{References}

1. EBIHARA T. (1981) Shear properties of laminatedveneer lumber. Mokuzai Gakkaishi, (27) 11, 788-794

2. ECHOLS R.M., CURRIER R.A. (1973) Comparative properties of Douglas-Fir made from Parallel-Laminated Veneers vs Solid Wood. Forest Products Journal, (23) 2, 45-47.

3. NF B51-008 (1987) Wood. Static bending. Determination of ultimate strength in static bending using small clear specimens", November 1987, 8 p.

4. NF B51-016 (1987) Wood. Static bending. Determination of modulus of elasticity in static bending using small clear specimens, December 1987, 8 p.

5. HOOVER W.L., RINGE J.M., ECKELMAN C.A., YOUNGQUIST J.A. (1987a) Material design factors for hardwood laminated-veneer-lumber. Forest Products Journal, (37) 9, 15-23

6. KILIC Y., COLAK N. BAYSAL E., BURDURLU E. (2006) An investigation of some physical and mechanical properties of laminated veneer lumber manufactured from black alder (Alnus glutinosa) glued with polyvinyl acetate and polyurethane adhesives. Forest Products Journal 56 (9), 56-59

7. KUNESH R.H. (1978) Micro-Lam: structural Laminated Veneer Lumber. Forest Products Journal, (28) $7,41-44$
8. LEI Y K. ET WILSON J.B. (1979) Fracture toughness of parallel-laminated veneer. Forest Products Journal, (29) 8, 28-31

9. MORIIZUMI, S.; MAEDA, N.; SANADA, Y.; OGURA, T. (1984) The strength properties of Japanese larch LVL (I). The wood quality of small Japanese larch logs and the bending strength of clear LVL Journal of the Hokkaido. Forest Products Research Institute $1984 n^{\circ}$ 384, 1-7

10. PAŁUBICKI B., MARCHAL R., BUTAUD J.-C., DENAUD L.-E., BLÉRON L., COLLET R., KOWALUK G. (2009). A Method of Lathe Checks Measurement; SMOF device and its software. In press, Wood Science and Technology.

11. SCHAFFER E.L., JOKERST R.W., MOODY R.C., PETERS C.C., TSCHERNITZ J.L. ET ZAHN J.J. (1972), Feasibility of producing a high-yield laminated sructural product : General Summary. Research paper FPL $\mathrm{n}^{\circ} 175,18 \mathrm{p}$.

12. THIBAUT B. (1988) Le processus de coupe du bois par déroulage. These de doctorat d'Etat, University of Montpellier, $386 \mathrm{p}$. 